Eur. J. Clin. Chem. Clin. Biochem.

Vol. 32, 1994, pp. 27-29

(c) 1994 Walter de Gruyter \& Co. Berlin - New York

\title{
Determination of Oxalate Excretion in Spot Urines of Healthy Children by Ion Chromatography
}

\author{
By Ch. von Schnakenburg ${ }^{1}$, D. J. Byrd ${ }^{1}$, K. Latta ${ }^{1}$, G. S. Reusz ${ }^{2}$, Dorothee Graf ${ }^{1}$ and J. Brodehl ${ }^{1}$ \\ 1 Laboratorium für pädiatrische Nieren- und Stoffwechselkrankheiten der Kinderklinik \\ der Medizinischen Hochschule Hannover, Hannover, Germany \\ ${ }^{2}$ First Department of Pediatrics, Semmelweis University Medical School, Budapest, Hungary
}

(Received July 13/October 26, 1993)

Summary: Evidence for the suitability of spot urines for selective screening in children was obtained by comparing the 24-hour urinary oxalate excretion with the ratio of urinary oxalate to creatinine $[\mathrm{mmol} / \mathrm{mol}]$ in spontaneously voided urine samples. Spot urines of 169 healthy children aged 1 day to 13 years were analysed in order to establish reference values for the urinary oxalate/creatinine ratio in relation to age and body surface area. Oxalate was measured by automated ion chromatography. Results showed an inverse relationship between the oxalate/creatinine ratio and age. The highest ratios, $131 \pm 57 \mathrm{mmol} / \mathrm{mol}$ (mean $\pm 2 \mathrm{SD}$ ), were found in infants. At age two years, the ratio was $84 \pm 55$, at age five years $56 \pm 35$, and for children older than ten years $42 \pm 31$. This finding can be explained by the gain of muscle mass and hence increased creatinine production with increasing age. Data for the urinary oxalate/creatinine ratio are presented according to body surface area for the assessment of children with abnormal growth. In 19 urine samples from nine patients with primary hyperoxaluria, the oxalate/creatinine ratio greatly exceeded $(286-2022 \mathrm{mmol} / \mathrm{mol})$ the above reference ranges. We therefore propose the determination of the oxalate/creatinine ratio in spot urines for the selective screening for hyperoxaluria in children with nephrocalcinosis or urolithiasis.

\section{Introduction}

Although nephrocalcinosis and urolithiasis are infrequent problems in children, early diagnosis and treatment are of critical importance to the outcome. The percentage of detectable metabolic abnormalities in these children increases up to $30 \%$ with the accuracy and intensity of laboratory examinations (1). In the past, mainly due to methodological problems, little attention has been paid to the role of oxalate as a factor in the genesis of urolithiasis and nephrocalcinosis. However, changes of urinary oxalate concentration have a 10-fold higher influence on the solubility of calcium oxalate than comparable changes of calcium concentration (2). Therefore, a meticulous search for abnormalities in oxalate metabolism is recommended in patients presenting with nephrocalcinosis and/or urolithiasis. Hyperoxaluria may be caused by hyperabsorption (3), or by increased endogenous production of oxalate as in primary hyperoxaluria type 1 or 2 (4).
This study presents results on the suitability of spot urines for selective oxalate screening in children. Urinary oxalate was determined by automated ion chromatography. We present reference values for urinary oxalate in relation to urinary creatinine, according to age and body surface area. Results from patients with primary hyperoxaluria are compared with these reference values.

\section{Subjects and Methods}

Healthy probands

Pre-noon spot urines of 169 apparently healthy children (95 male, 74 female, age one day to thirteen years) were collected on a maternity ward, in a kindergarten and in a school. Spot urines and 24hour urines from 15 children between 3 and 15 years (mean 10), who were hospitalized for reasons not related to oxalate metabolism, were investigated to determine the relationship between the 24-hour oxalate excretion and the oxalate/creatinine ratio in spot urines. 
Pationts

Spot urines were collected from 9 patients with primary hyperoxaluria, aged between 0.5 and 30 years (mean 8.4). In two of these cascs, the glomerular filtration rate was less than $20 \mathrm{ml} / \mathrm{min}$ per $1.73 \mathrm{~m}^{2}$. in three others it was between 20 and 60 , while it was over 60 in the remaining four. A 24-hour urine collection was performed on four of these patients.

Height and weight were recorded in all probands and used for calculation of body surface area.

\section{Sample handling}

After collection urine samples were immediately acidified by adding $0.1 \mathrm{ml}$ of $6 \mathrm{~mol} / 1$ hydrochloric acid to $5 \mathrm{ml}$ aliquots (5). These samples were then diluted 20 -fold with $0.3 \mathrm{~mol} / / \mathrm{l}$ boric acid and stored at $-20^{\circ} \mathrm{C}(6)$.

\section{Ion chromatography}

Oxalate was separated and quantified, using an automated ion chromatography system (Sykam, Gilching, Germany) with Dionex (Idstein, Germany) columns and suppressor unit (AG4A, AS4A, AMMS2), in combination with a Marathon autosampler (Spark, Friedrichsdorf, Germany). The procedure was a modified version of a previously described method (7). Oxalate was calculated by the peak height method after calibration with an acidified external standard (Shimadzu integrator CR5A). Results from ion chromatography (IC) correlated well with those of a gas chromatographic (GC) method (8) formerly used routinely in our laboratory: $y(\mathrm{IC})$ $[\mu \mathrm{mol} / \mathrm{l}]=0.87 \times(\mathrm{GC})+31.6\left(\mathrm{R}^{2}=0.96, \mathrm{p}<0.01, \mathrm{n}=22\right.$, concentration range 10 to $700 \mu \mathrm{mol} / \mathrm{l})$. Our laboratory participates successfully in an international external quality assurance scheme for urinary oxalate (University College London). Day-to-day precision in 20 aliquots of a urine sample was $3.4 \%$ over six months (oxalate concentration $338 \mu \mathrm{mol} / \mathrm{l})$ and intra-run precision was $1 \%$ (20 determinations). The average recovery was $95.4 \%$ (SD 5.7) over a range of added oxalate of 14 to $2221 \mu \mathrm{mol} / \mathrm{l}$. Creatinine was measured with the help of the creatinine analyzer 2 (Beckman, München, Germany).

\section{Results}

In 169 spot urines of healthy non-hospitalized children the oxalate/creatinine ratio $[\mathrm{mmol} / \mathrm{mol}]$ ranged from 24.8 to 195 (mean 61.1). The highest mean ratio was found during the neonatal period, and it decreased rapidly with increasing age (fig. 1). Similar inverse and

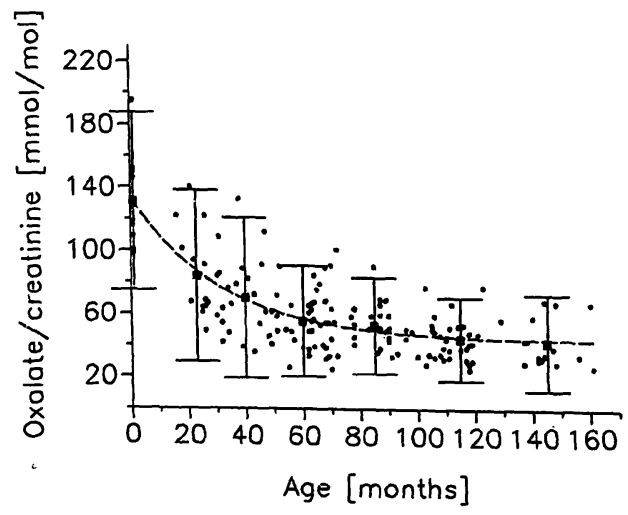

Fig. 1 Relationship between urinary oxalate/creatinine ratio and age in 169 healthy infants and children: the regression curve is depicted by $y=90.8 \mathrm{e}^{-0.031 \mathrm{x}}+43.1\left(\mathrm{R}^{2}=0.587\right)$; means $\pm 2 \mathrm{SD}$ are presented according to the data in table 1 . non-linear correlations of the oxalate/creatinine ratio to body surface area, body weight and height were found ( $\mathrm{R}^{2}$ ranges between 0.582 and 0.593 ). The data in relation to age and body surface area are presented in table 1. In urine samples of the patients with primary hyperoxaluria, the oxalate/creatinine ratio ranged from 286 to $2022 \mathrm{mmol} / \mathrm{mol}$ (mean 735 , SD $464, \mathrm{n}=19$ ) and was therefore distinctly higher than our reference intervals.

A correlation between oxalate excretion in 24 hours per $1.73 \mathrm{~m}^{2}$ and the oxalate/creatinine ratio in spot urines was found in 15 healthy children (fig. 2) and in 4 patients with primary hyperoxaluria. In healthy children the regression is described by y $\left[\mu \mathrm{mol} / \mathrm{d} \times 1.73 \mathrm{~m}^{2}\right]$ $=7.7 \mathrm{x}+5[\mathrm{mmol} / \mathrm{mol}]\left(\mathrm{R}^{2}=0.528, \mathrm{p}<0.01\right)$, while

Tab. 1 Urinary oxalate/creatinine ratio in relation to age and body surface area of 169 healthy children (means $\pm 2 \mathrm{SD}$ ).

\begin{tabular}{ccc}
\hline Age [months] & $\mathrm{n}$ & $\begin{array}{l}\text { Oxalate/creatinine-ratio } \\
{[\mathrm{mmol} / \mathrm{mol}]}\end{array}$ \\
\hline$<1$ & 11 & $131(75-188)$ \\
$16-30$ & 15 & $84(29-139)$ \\
$31-50$ & 23 & $70(20-121)$ \\
$51-70$ & 39 & $56(20-91)$ \\
$71-100$ & 32 & $52(22-83)$ \\
$101-130$ & 34 & $44(18-71)$ \\
$131-161$ & 15 & $42(12-73)$
\end{tabular}

Body surface area $\left[\mathrm{m}^{2}\right]$

\begin{tabular}{rrr}
$0.16-0.24$ & 11 & $131(75-188)$ \\
$0.40-0.60$ & 11 & $82(25-139)$ \\
$0.61-0.70$ & 23 & $73(23-123)$ \\
$0.71-0.80$ & 21 & $60(14-107)$ \\
$0.81-0.90$ & 23 & $59(23-95)$ \\
$0.91-1.00$ & 22 & $50(21-79)$ \\
$1.01-1.20$ & 32 & $45(23-67)$ \\
$1.21-1.50$ & 24 & $46(16-76)$ \\
$>1.60$ & 2 & 29 and 26 \\
\hline
\end{tabular}

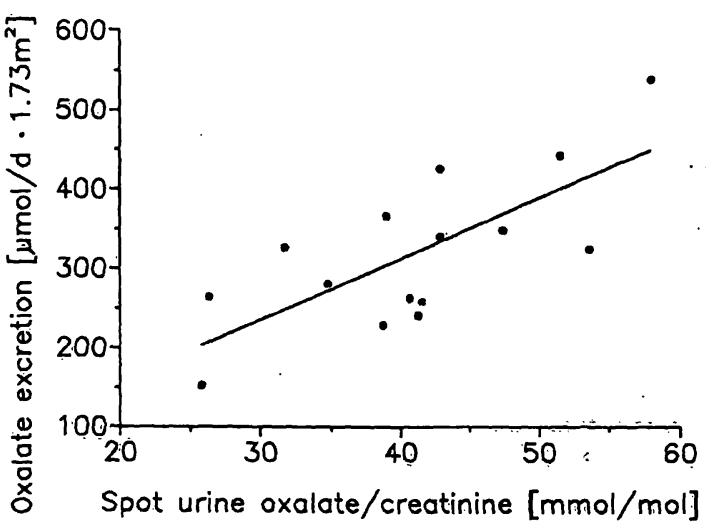

Fig. 2 Relationship between urinary oxalate excretion per day per $1.73 \mathrm{~m}^{2}$ and the oxalate/creatinine ratio in spot urines of 15 healthy children $\left(y=7.7 x+5 ; R^{2}=0.528\right)$; 
in the 4 hyperoxaluric subjects a correlation of $y=11.2 x-433\left(R^{2}=0.799, p<0.01\right)$ was derived from 9 separate collections.

\section{Discussion}

In children with urinary stones and/or nephrocalcinosis, various tests must be performed to establish the metabolic causes, including the determination of urinary oxalate. To the best of our knowledge, this is the first study to present reference ranges for urinary oxalate in healthy non-hospitalized children, determined by ion chromatography. The method is reliable, precise and convenient (9).

In our hands results obtained with ion chromatography were closely related to those determined by a reference gas chromatographic method. The highest oxalate/creatinine ratio was found in infants. The ratio decreased markedly with age, due to the gain in muscle mass and the resulting increase in creatinine production. The agerelated reference ranges agree with results from studies using an 1 . $(10,11)$. Oxalate excretion corrected for body surface area is not age-dependent (12).

Our data demonstrated a good correlation for timed urines and spot urines. These results are in accordance with another smaller study demonstrating the accuracy of spot urine screening for primary hyperoxaluria, in

\section{References}

1. Laufer, J. \& Boichis, H. (1989) Urolithiasis in children: Current medical management. Pediatr. Nephrol. 3, 317-331.

2. Berg, W. (1990) Stoffwechsel und Pathophysiologie der Oxalsäure. Z. Urol. Nephrol. 83, 481-488.

3. Rampton, D. S. \& Scarner, M. (1988) Enteric and other secondary hyperoxalurias. In: Oxalate Metabolism in Relation to Urinan, Stone (Rose, G. A., ed.) pp. 103-120, Springer, London.

4. Hillman, R. E. (1989) Primary hyperoxalurias. In: The Metabolic Basis of Inherited Diseases, 6 th edn. (Scriver, C. R., Beaudet, A. L., Sly, W. S. \& Valle, D., eds.) pp. 933-944, McGraw-Hill, New York.

5. Hodgkinson, A. (1981) Sampling errors in the determination of urine calcium and oxalate: Solubility of calcium oxalate in HCl-urine mixtures. Clin. Chim. Acta 109, 239-244.

6. Robertson, W. G. \& Scurr, D. S. (1984) Prevention of ascorbic acid interference in the measurement of oxalic acid in urine by ion-chromatography. Clin. Chim. Acta 140, 97-99.

7. Robertson, W. G., Scurr, D. S., Smith, A. \& Orwell, R. L. (1982) The determination of oxalate in urine and urinary calculi by a new ion-chromatographic technique. Clin. Chim. Acta 126, 91-99.

8. Farrington, C. J. \& Chalmers, A. H. (1979) Gas-chromatographic estimation of urinary oxalate and its comparison with a colorimetric method. Clin. Chem. 25, 1993-1996.

9. Classen, A. \& Hesse, A. (1987) Measurement of urinary oxalate: An enzymatic and an ion chromatographic method compared. J. Clin. Chem. Clin. Biochem. 25, 95-99. which a single patient had a clearly higher value than five healthy controls (13).

Especially in small children and infants, timed urines are difficult to collect with any degree of confidence. For screening purposes, we therefore recommend determination of the urinary oxalate/creatinine ratio in spot urines. However, if an elevated urinary oxalate/creatinine ratio is found, the analysis should be repeated, so that alimentary origins (e. g. spinach, chocolate) or secondary causes (3) for the elevated ratio can be ruled out prior to the diagnosis of primary hyperoxaluria. Further urinary analyses or enzyme determinations (e.g. in liver biopsies) should be subsequently performed to classify the exact type of hyperoxaluria (14).

Renal failure complicates the diagnosis of hyperoxaluric subjects. It has been demonstrated that oxalate clearance decreases more rapidly than the glomerular filtration rate as renal failure progresses (15). Therefore, oxalate excretion may be normal or almost normal in affected individuals with renal failure. In contrast, due to the simultaneous reduction of the urinary creatinine concentration with renal failure, the oxalate/creatinine ratio will remain high, and will still be useful for the diagnosis of hyperoxaluria.

We conclude that urinary oxalate/creatinine ratios in spot urines are a helpful screening tool for children in all age groups in the search for possible causes of nephrolithiasis or nephrocalcinosis.

10. Barratt, T. M., Kasidas, G. P., Murdoch, I. \& Rose, G. A. (1991) Urinary oxalate and glycolate excretion and plasma oxalate concentration. Arch. Dis. Child. 66, 501-503.

11. Leumann, E. P., Dietl, A. \& Matasovic, A. (1990) Urinary oxalate and glycolate excretion in healthy infants and children. Pediatr. Nephrol. 4, 493-497.

12, Gibbs, D. A. \& Watts, R. W. E. (1969) The variation of urinary oxalate excretion with age. J. Lab. Clin. Med. 73, 901-908.

13. Itami, N., Yasoshima, K., Akutsu, Y. \& Nonomura, K. (1990) Spot-urine screening for primary hyperoxaluria. Nephron 56 , 337-338.

14. Danpure, C. J., Jennings, P. R. \& Watts, R. W. E. (1987) Enzymological diagnosis of primary hyperoxaluria type 1 by measuring the alanine : glyoxylate aminotransferase activity in hepatic percutaneous needle biopsies. Lancet $1,289-291$.

15. Morgan, S. H., Purkiss, P., Watts, R. W. E. \& Mansell, M. A. (1987) Oxalate dynamics in chronic renal failure. Nephron 46, 253-257.

Dr. D. J. Byrd

Laboratorium für pädiatrische Nierenund Stoffwechselkrankheiten

Kinderklinik

Medizinische Hochschule Hannover

D-30623 Hannover

Germany 
